\title{
Diagnóstico nutrimental en lima 'Persa' (Citrus latifolia Tanaka) con análisis estadístico por componentes principales
}

\author{
Nutrimental diagnosis in 'Persian' lime (Citrus latifolia Tanaka) with statistical analysis \\ using principal components
}

Fredy Nieves-González ${ }^{1}$, Gelacio Alejo-Santiago*, ${ }^{2 *}$ Porfirio Juárez-López ${ }^{1}$, Gregorio Luna-Esquivel²

\begin{abstract}
RESUMEN
El objetivo fue identificar la interacción de la materia orgánica $(\mathrm{MO}), \mathrm{pH}$, disponibilidad nutrimental en suelo y concentración en tejido vegetal de nitrógeno $(\mathrm{N})$, fósforo $(\mathrm{P})$, potasio $(\mathrm{K})$, calcio $(\mathrm{Ca})$ y magnesio $(\mathrm{Mg})$ en huertas de lima 'Persa' (Citrus latifolia Tanaka), a través del método de componentes principales. Se realizaron muestreos de suelo y foliares en 30 huertos de lima 'Persa' en la zona productora del estado de Nayarit (municipios de Xalisco, Tepic, Santa María del Oro y San Pedro Lagunillas), en 2015. Por medio del análisis estadístico por componentes principales, se agruparon 12 variables (en suelo: $\mathrm{pH}, \mathrm{MO}$, nitrógeno disponible anual, $\mathrm{P}, \mathrm{K}, \mathrm{Ca}$ y $\mathrm{Mg}$; en tejido vegetal: $\mathrm{N}, \mathrm{P}, \mathrm{K}, \mathrm{Ca}$ y Mg). Los resultados indicaron que, con excepción de nitrógeno en tejido vegetal $(\mathrm{N} \mathrm{tv})$, las variables se agruparon en dos componentes principales y representaron $86.73 \%$ de la varianza. El primer componente correlacionó fuertemente variables de carácter químico-orgánicas y, el segundo, variables de tipo físico-químicas. Se observaron seis grupos con características similares en cuanto al estado nutrimental. Este método estadístico aportó una idea visual de las interacciones entre las variables y permite realizar acciones inmediatas conforme a las necesidades nutrimentales de cada huerto.
\end{abstract}

PALABRAS CLAVE

análisis multivariado, agricultura sustentable, interacción nutrimental visual

\begin{abstract}
The objective of this study was to identify the interaction of organic matter $(\mathrm{OM}), \mathrm{pH}$, nutritional availability in soil and concentration in plant tissue of nitrogen $(\mathrm{N})$, phosphorus $(\mathrm{P})$, potassium $(\mathrm{K})$, calcium $(\mathrm{Ca})$, and magnesium $(\mathrm{Mg})$ in Persian lime (Citrus latifolia Tanaka) orchards, through the principal components method. Soil and leaf samples were collected in 30 Persian lemon orchards in the Mexican production area of Nayarit (Xalisco, Tepic, Santa Maria del Oro, and San Pedro Lagunillas municipalities), in 2015. A statistical analysis of main components was performed with 12 variables (soil $\mathrm{pH}$, OM, nitrogen available annually, phosphorus, potassium, calcium and magnesium, plant tissue nitrogen, phosphorus, potassium, calcium and magnesium). The results indicate that, with exception of nitrogen in plant tissue ( $\mathrm{N} \mathrm{tv})$, the variables were grouped into two components and represented $86.73 \%$ of the variance. The first component strongly correlated chemical-organic variables and the second component physical-chemical variables. Six groups with similar characteristics were observed regarding nutritional status. This statistical method provides a visual idea of the interactions between the variables and allows immediate actions according to the nutritional needs of each orchard.
\end{abstract}

KEYWORDS

multivariate analysis, sustainable agriculture, visual nutritional interaction

\footnotetext{
${ }^{1}$ Facultad de Ciencias Agropecuarias, Universidad Autónoma del Estado de Morelos, Cuernavaca, Morelos, México.

${ }^{2}$ Unidad Académica de Agricultura, Universidad Autónoma de Nayarit, Nayarit, México.

*Autor para correspondencia. Carretera Tepic-Compostela, km 9. 63780, Xalisco, Nayarit, México.

Correo electrónico: gelacioalejo@hotmail.com
} 


\section{INTRODUCCIÓN}

Los métodos estadísticos son una herramienta fundamental para interpretar y evaluar los resultados de investigaciones científicas. $\mathrm{Al}$ respecto, el método de componentes principales permite agrupar gran cantidad de variables, asociarlas y representarlas en un gráfico cuyas coordenadas indican la correlación de las variables con los factores y, con ello, agrupar las variables con relación a su interacción y características propias (Camacho et al., 2010; Castañón et al., 2008).

Este método puede dar una visión de la interacción de variables importantes de fertilidad del suelo (Campitelli et al., 2010) y la disponibilidad nutrimental mediante la concentración de los nutrimentos en el tejido vegetal de los cultivos (Etchevers, 1999). En lima 'Persa', Citrus latifolia Tanaka (Rutaceae), se han reportado concentraciones nutrimentales de biomasa seca. En éstas, se ha clasificado y correlacionado, con apoyo de indicadores de concentración foliar (Contreras et al., 2007; Maldonado et al., 2008), la disponibilidad de algunos macronutrimentos en el suelo con la calidad del fruto (Mattos et al., 2010). No obstante, las interacciones entre la disponibilidad y la concentración de nutrimentos y parámetros del suelo que alteran el estado nutrimental de huertas en producción, las cuales cumplen un papel importante para la economía mexicana, no se han medido. Lo anterior se debe a que México es el segundo productor mundial de lima 'Persa' (a la que también se le conoce como limón 'Persa'), con una superficie sembrada de $180,601.98$ ha, equivalentes a $10,326,251,980.00$ pesos mexicanos, de los cuales, Nayarit aporta 2,363.32 ha, que representan $101,938,320.00$ pesos mexicanos (SIAP, 2017).

El objetivo de este estudio fue identificar la interacción de la materia orgánica $(\mathrm{MO}), \mathrm{pH}$, disponibilidad nutrimental en suelo y concentración en tejido vegetal de nitrógeno $(\mathrm{N})$, fósforo $(\mathrm{P})$, potasio $(\mathrm{K})$, calcio (Ca) y magnesio (Mg) en huertas de lima 'Persa' a través del método de componentes principales.

\section{Materiales y mÉtodos}

La recolección de muestras se realizó en huertas de lima 'Persa' de aproximadamente 12 años, ubicadas en los municipios de Xalisco (26 muestras), Tepic (30 muestras), Santa María del Oro (14 muestras) y San Pedro Lagunillas (12 muestras) con intervalos de altura de 750-1,250 m. En total, se realizaron 82 muestreos (41 de suelo y 41 de tejido vegetal).
A las muestras de suelo se les determinó materia orgánica $(\mathrm{MO})$ por el método de oxidación de carbono orgánico con dicromato de potasio (Walkey y Black, 1947); $\mathrm{pH}$ con relación agua-suelo $2: 1 ; \mathrm{N}$ por la fracción mineralizable anual de la materia orgánica; el P por el método de Bray-Kurts (1945) y K, Ca y Mg por lavado de suelo con acetato de amonio.

El muestreo de tejido vegetal se realizó en ramas sin fruto con hojas maduras del flujo de crecimiento anterior (Maldonado et al., 2008). Las muestras (hojas del ciclo anterior) se procesaron y analizaron en el laboratorio de Análisis de Suelo, Agua y Planta de la Unidad Académica de Agricultura de la Universidad Autónoma de Nayarit.

Las muestras de tejido vegetal se colocaron en bolsas de papel y se deshidrataron en estufa de desecación a $60^{\circ} \mathrm{C}$ hasta alcanzar peso constante. Posteriormente, se trituraron con molino eléctrico marca $\mathrm{IKA}^{\circledR}$ modelo A11 basic. Se procesaron por medio de digestión ácida. La determinación de $\mathrm{N}$ se efectuó por digestión ácida y arrastre de vapor (micro Kjeldahl); $\mathrm{P}$ por colorimetría con el método de amarillo de vanadato-molibdato; $\mathrm{K}$ por flamometría, $\mathrm{Ca}$ y $\mathrm{Mg}$ con absorción atómica.

\section{Análisis estadístico}

Se probó normalidad mediante la prueba de Kolmogorov-Smirnov; las variables que no presentaron normalidad $(p<0.20)$ se transformaron a logaritmo natural. Posteriormente, el total de las variables se estandarizaron a Z. Después se les aplicó el análisis de componentes principales. Se utilizó el programa estadístico STATISTICA 13.

\section{RESULTADOS Y DISCUSIóN}

En el cuadro 1 se presentan las correlaciones entre las variables evaluadas. La materia orgánica (MO) correlacionó fuertemente $(p>0.01)$ con nitrógeno en suelo (N s), debido a que el cálculo de nitrógeno en suelo (N s) se realizó a partir de la mineralización de la MO (Boechat et al., 2013). A su vez, las correlaciones de $\mathrm{MO}$ con Ca y Mg tanto en suelo como en la planta, fueron fuertemente negativas $(p>0.01), y$ con potasio en tejido vegetal $(\mathrm{K} t v)$ fue fuertemente positiva $(p>$ $0.01)$.

El $\mathrm{pH}$ presentó correlaciones positivas $(p>0.05)$ con la mayoría de cationes contenidos en suelo y planta, excepto con potasio en tejido vegetal (K tv). Presentó también una correlación positiva $(p>0.05)$ con fósforo 
Cuadro 1. Correlaciones de las variables en muestras de suelo y tejido vegetal en lima 'Persa' (Citrus latifolia Tan.).

\begin{tabular}{|c|c|c|c|c|c|c|c|c|c|c|c|c|}
\hline & $M O$ & $p H$ & $N s$ & $N t v$ & $P s$ & $P$ tv & $K s$ & Ktv & Cas & Catv & $M g s$ & $M g t v$ \\
\hline MO & 1.00 & & & & & & & & & & & \\
\hline$p H$ & -0.49 & 1.00 & & & & & & & & & & \\
\hline$N s$ & 1.00 & -0.49 & 1.00 & & & & & & & & & \\
\hline$N t v$ & 0.39 & -0.20 & 0.39 & 1.00 & & & & & & & & \\
\hline$P s$ & -0.67 & -0.31 & -0.67 & -0.41 & 1.00 & & & & & & & \\
\hline$P$ tv & -0.99 & 0.48 & -1.00 & -0.36 & 0.67 & 1.00 & & & & & & \\
\hline$K s$ & -0.16 & 0.94 & -0.16 & -0.08 & -0.61 & 0.15 & 1.00 & & & & & \\
\hline$K t v$ & 0.87 & -0.35 & 0.87 & -0.11 & -0.56 & -0.89 & -0.05 & 1.00 & & & & \\
\hline Cas & -0.84 & 0.81 & -0.84 & -0.07 & 0.18 & 0.85 & 0.58 & -0.83 & 1.00 & & & \\
\hline Ca tv & -0.99 & 0.50 & -1.00 & -0.46 & 0.66 & 0.99 & 0.18 & -0.83 & 0.83 & 1.00 & & \\
\hline$M g s$ & -0.59 & 0.85 & -0.59 & 0.21 & -0.16 & 0.60 & 0.73 & -0.69 & 0.93 & 0.56 & 1.00 & \\
\hline$M g$ to & -0.82 & 0.53 & -0.82 & -0.83 & 0.53 & 0.80 & 0.28 & -0.43 & 0.59 & 0.86 & 0.31 & 1.00 \\
\hline
\end{tabular}

$\mathrm{MO}=$ materia orgánica; $\mathrm{N}$ s= nitrógeno en suelo; $\mathrm{N}$ tv= nitrógeno en tejido vegetal; $\mathrm{P} \mathrm{s}=$ fósforo en suelo; $\mathrm{P}$ tv= fósforo en tejido vegetal; $\mathrm{K} \mathrm{s}=$ potasio en suelo; $\mathrm{K}$ tv= potasio en tejido vegetal; $\mathrm{Ca}$ s= calcio en suelo; $\mathrm{Ca}$ tv= calcio en tejido vegetal; $\mathrm{Mg}$ s= magnesio en suelo; $\mathrm{Mg}$ tv= magnesio en tejido vegetal.

en tejido vegetal ( $\mathrm{P}$ tv) y negativa $(p>0.05)$ con fósforo en suelo (P s) y nitrógeno en suelo ( $\mathrm{N} \mathrm{s})$.

Se observaron correlaciones fuertemente negativas $(p>0.01)$ de nitrógeno en suelo ( $\mathrm{N} \mathrm{s}$ ) con fósforo en suelo (P s), fósforo en tejido vegetal (P tv), calcio en suelo (Ca s), calcio en tejido vegetal (Ca tv), magnesio en suelo (Mg s) y magnesio en tejido vegetal ( $\mathrm{Mg} \mathrm{tv})$; presentó correlaciones fuertemente positivas $(p>0.01)$ con potasio en tejido vegetal $(\mathrm{K} t v)$ y positivas $(p>0.05)$ con nitrógeno en tejido vegetal $(\mathrm{N} \mathrm{tv})$.

Nitrógeno en tejido vegetal $(\mathrm{N}$ tv) presentó fuerte correlación negativa $(p>0.01)$ con magnesio en tejido vegetal $(\mathrm{Mg} \mathrm{tv})$. Fósforo en suelo (P s) correlacionó negativamente $(p>0.05)$ con potasio en suelo $(\mathrm{K} s)$, potasio en tejido vegetal $(\mathrm{K} \mathrm{tv})$ y positivamente $(p>0.05)$ con magnesio en tejido vegetal $(\mathrm{Mg} \mathrm{tv})$.

Fósforo en tejido vegetal (P tv) correlacionó negativamente $(p>0.05)$ con potasio en tejido vegetal (K tv) y positivamente $(p>0.05)$ con el resto de los cationes contenidos en suelo y planta. Potasio en suelo (K s) está positivamente $(p>0.05)$ correlacionado con los demás cationes. Potasio en tejido vegetal $(\mathrm{K} \mathrm{tv}) \mathrm{co}-$ rrelacionó negativamente $(p>0.05)$ con los cationes contenidos en suelo y planta. Calcio en suelo (Ca s) correlacionó fuertemente negativo $(p>0.01)$ con calcio en tejido vegetal (Ca tv) y magnesio en suelo ( $\mathrm{Mg} \mathrm{s}$ ) y negativa $(p>0.05)$ con magnesio en tejido vegetal $(\mathrm{Mg} \mathrm{tv})$. Calcio en tejido vegetal (Ca tv) correlacionó fuertemente positivo $(p>0.01)$ con magnesio en tejido vegetal (Mgtv) y con magnesio en suelo (Mgs). Magnesio en suelo $(\mathrm{Mg} s)$ presentó correlación positiva $(p>0.05)$ con magnesio en tejido vegetal ( $\mathrm{Mg} \mathrm{tv})$.

En el cuadro 2 se presenta el porcentaje de varianza que corresponde a cada factor. Se observa que, con dos factores, arrojados por el análisis de componentes principales, se explica más del $85 \%$ de la varianza de las doce variables analizadas.

Cuadro 2. Varianza y porcentaje acumulativo de los factores en el análisis de componentes principales.

\begin{tabular}{|c|c|c|c|c|}
\hline FACTOR & $\begin{array}{l}\text { A U T O - } \\
\text { VALORES }\end{array}$ & $\begin{array}{c}\% \text { Total } \\
\text { DE } \\
\text { VARIANZA }\end{array}$ & $\begin{array}{c}\text { Auto- } \\
\text { VAlORES } \\
\text { ACUMULAdo }\end{array}$ & $\begin{array}{l}\% \text { VARIANZA } \\
\text { ACUMUlado }\end{array}$ \\
\hline 1 & 7.51 & 62.63 & 7.51 & 62.63 \\
\hline 2 & 2.89 & 24.09 & 10.40 & 86.72 \\
\hline 3 & 1.59 & 13.27 & 12.00 & 100 \\
\hline
\end{tabular}

En el cuadro 3 se presenta la correlación de las variables con los factores. Con el factor 1 correlacionan positivamente materia orgánica $(\mathrm{MO})$, nitrógeno en suelo $(\mathrm{N} \mathrm{s})$ y potasio en tejido vegetal $(\mathrm{K} \mathrm{tv})$ y negativamente fósforo en tejido vegetal $(\mathrm{P} \mathrm{tv})$, calcio en suelo (Ca s), calcio en tejido vegetal (Ca tv), magnesio en suelo (Mg s) y magnesio en tejido vegetal ( $\mathrm{Mg} \mathrm{tv})$. Con el factor 2 correlacionó positivamente fósforo en suelo $(\mathrm{P} \mathrm{s})$ y negativamente $\mathrm{pH}$ y potasio en suelo 
Cuadro 3. Correlación entre los factores del análisis de Componentes principales y variables de suelo y tejido vegetal en lima 'Persa' (Citrus latifolia).

\begin{tabular}{ccc}
\hline VARIABLE & $\begin{array}{l}\text { INTERACCIONES } \\
\text { Q U Í M I C O } \\
\text { ORgÁNICAs } \\
\text { (FACTOR 1) }\end{array}$ & $\begin{array}{c}\text { INTERACCIONES } \\
\text { Físico- } \\
\text { QuímicAs } \\
\text { (FACTOR 2) }\end{array}$ \\
\hline$M O$ & 0.98 & -0.18 \\
$p H$ & -0.65 & -0.71 \\
$N s$ & 0.98 & -0.18 \\
$N$ tv & 0.37 & -0.38 \\
$P s$ & -0.50 & 0.85 \\
$P t v$ & -0.97 & 0.18 \\
$K s$ & -0.35 & -0.87 \\
$K t v$ & 0.84 & -0.07 \\
$C a s$ & -0.91 & -0.35 \\
$C a t v$ & -0.98 & 0.19 \\
$M g s$ & -0.70 & -0.65 \\
$M g t v$ & -0.82 & 0.20 \\
\hline
\end{tabular}

$\mathrm{MO}=$ materia orgánica; $\mathrm{N} \mathrm{s}=$ nitrógeno en suelo; $\mathrm{N}$ tv $=$ nitrógeno en tejido vegetal; $\mathrm{P} \mathrm{s}=$ fósforo en suelo; $\mathrm{P} \mathrm{tv}=$ fósforo en tejido vegetal; $\mathrm{K} \mathrm{s}=$ potasio en suelo; $\mathrm{K} \mathrm{tv}=$ potasio en tejido vegetal; $\mathrm{Ca} \mathrm{s}=$ calcio en suelo; $\mathrm{Ca} \mathrm{tv}=$ calcio en tejido vegetal; $\mathrm{Mg} \mathrm{s}=$ magnesio en suelo; $\mathrm{Mg}$ tv= magnesio en tejido vegetal.

$(\mathrm{K} \mathrm{s})$. nitrógeno en tejido vegetal $(\mathrm{N}$ tv) no presentó correlaciones significativas con ambos factores.

La figura 1 indica la ubicación de las muestras con relación a los factores. En el primer cuadrante se ubican 13 muestras; en el segundo, diez; en el tercer cuadrante, tres; en el cuarto cuadrante, 15; con un total de 41 muestras. De igual manera, esta figura permite agrupar las muestras con relación a la situación nutrimental en que se encuentran. Se observan seis agrupaciones rodeadas con las líneas punteadas.

Las correlaciones de las variables con el factor 1 indican fuertes interacciones de los componentes orgánicos del suelo con la disponibilidad de los nutrimentos y el contenido de los mismos en el tejido vegetal. Debido a estas interacciones este factor se etiquetó como interacciones químico-orgánicas (cuadro 3).

Dentro de estas interacciones destaca la correlación positiva de materia orgánica $(\mathrm{MO})$ con nitrógeno en suelo $(\mathrm{N} \mathrm{s})$ y potasio en tejido vegetal $(\mathrm{K} \mathrm{tv})$. Se observa también una correlación fuertemente negativa de la materia orgánica (MO) con fósforo en tejido vegetal (P tv), calcio en suelo (Ca s), calcio en tejido vegetal (Ca tv), magnesio en suelo ( $\mathrm{Mg} \mathrm{s})$ y magnesio en tejido vegetal $(\mathrm{Mg} \mathrm{tv})$. Esto indica que el incremento

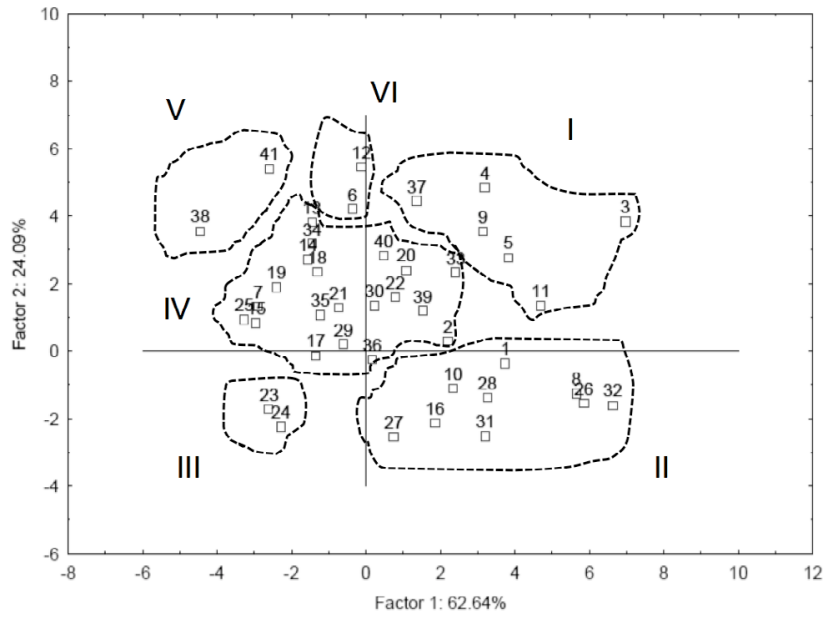

Figura 1. Ubicación de las muestras en relación a la interacción con los factores. El factor 1 representa las interacciones químico-orgánicas y el factor 2 las interacciones físicoquímicas.

de la materia orgánica (MO) en el suelo reduce la disponibilidad inmediata de calcio en suelo (Ca s) y magnesio en suelo ( $\mathrm{Mg} \mathrm{s}$ ) y la concentración de fósforo, calcio y magnesio en tejido vegetal ( $\mathrm{P} \mathrm{tv}, \mathrm{Ca}$ $\mathrm{tv}$ y $\mathrm{Mg} \mathrm{tv}$ ). Esto se observa en las correlaciones entre variables reportadas en el cuadro 1.

Estas correlaciones resultan congruentes al analizar los experimentos realizados por Bodin et al. (2006), Grigatti et al. (2012) y Castro et al. (2009), quienes observaron una rápida disponibilidad del $\mathrm{N}$ procedente de residuos orgánicos, pero una lenta disponibilidad de los otros macronutrimentos. Hussain et al. (2015) fertilizaron con compostas y obtuvieron disponibilidades de $\mathrm{P}$ y K similares a las de su tratamiento control. Aunque no se reportan los resultados para $\mathrm{Ca}$ y $\mathrm{Mg}$, el efecto bien podría ser similar debido a la alta cantidad de componentes orgánicos que retienen los nutrimentos en forma de iones, los cuales son liberados lenta y constantemente por acción de los microorganismos en las huertas (Julca et al., 2006).

Las variables que correlacionan con el factor 2 (cuadro 3) indican una interacción de propiedades físicas y químicas del suelo. Se consideró conveniente identificar este factor con el nombre interacciones físico-químicas. El $\mathrm{pH}$ y el potasio en suelo ( $\mathrm{K}$ s) presentan una correlación fuertemente negativa con el factor, mientras que fósforo en suelo ( $\mathrm{P} s$ ) presenta una correlación fuertemente positiva. Esto sugiere que una disminución del $\mathrm{pH}$ reduce la disponibilidad de potasio en suelo (K s) pero incrementa 
la disponibilidad de fósforo en suelo (P s). Ésta es una suposición contraria a lo que se ha observado en otras investigaciones (cuadro 1). La correlación entre MO y fósforo en suelo (P s) fue más fuerte que la correlación entre fósforo en suelo ( $\mathrm{P} \mathrm{s}$ ) y $\mathrm{pH}$, lo que indica que la disponibilidad de fósforo en suelo ( $\mathrm{P}$ s) está determinada por la MO más que por el $\mathrm{pH}$ del suelo. Esto se atribuye al efecto positivo en las propiedades físicas que un contenido adecuado de MO le aporta al suelo (Gallardo et al., 2009).

La variable nitrógeno en tejido vegetal $(\mathrm{N}$ tv) fue la única que no correlacionó significativamente con alguno de los componentes principales. Esta variable sólo presentó fuerte correlación negativa con magnesio en tejido vegetal ( $\mathrm{Mg} \mathrm{tv}$ ) (cuadro 1). Esto supone un antagonismo de estos elementos. Además, se observa que la cantidad de $\mathrm{N}$ acumulada en el tejido vegetal es constante y está poco influenciada por nitrógeno en suelo (N s) y MO. Se deduce, entonces, que las aportaciones de $\mathrm{N}$ a través de compostas y residuos orgánicos inocuos pueden reducir las pérdidas de $\mathrm{N}$ que se tienen con las fertilizaciones minerales.

La figura 1 permite agrupar las huertas estudiadas con base en las características predominantes de cada asociación de muestras. El grupo I presentan un pH muy ácido. Las muestras del grupo II presentan bajos contenidos de nitrógeno en tejido vegetal ( $\mathrm{N}$ tv) y magnesio en tejido vegetal ( $\mathrm{Mg} \mathrm{tv}$ ) de tal forma que, al alejarse hacia la derecha del eje del factor 2, la concentración de elementos disminuye. El grupo III presenta bajas concentraciones de nitrógeno en tejido vegetal $(\mathrm{N} t v)$ y fósforo en tejido vegetal $(\mathrm{P} \mathrm{tv})$. En el grupo IV se ubican las muestras con mejores condiciones nutrimentales en suelo y planta; no obstante, conforme los puntos de ubicación se alejan hacia la esquina superior izquierda, éstas presentan bajo contenido de materia orgánica (MO); cuando los puntos se van alejando hacia la esquina superior derecha, el pH se va tornando ácido. En el grupo V las muestras presentan muy bajo contenido de materia orgánica $\mathrm{MO}$, y el grupo VI presenta bajas concentraciones de magnesio en tejido vegetal $(\mathrm{Mg} \mathrm{tv})$.

Es notable la eficacia de este método en la caracterización nutrimental de las huertas y árboles de lima 'Persa' estudiados. Con esta información es posible realizar acciones correctivas en las huertas que presentan severos problemas nutrimentales.

La disponibilidad nutrimental del suelo y planta no deberían valorarse sólo por algún índice de concentración, ya que no indican ni permiten visualizar la interacción de los nutrimentos con factores químicos, físicos y orgánicos del suelo, ni su efecto directo e indirecto sobre el cultivo.

\section{ConClusiones}

El método estadístico de componentes principales permitió agrupar eficazmente las interacciones de las principales características nutrimentales de los suelos y tejido vegetal de lima 'Persa'. Con lo anterior, es posible identificar el estado nutrimental, lo que permitiría realizar acciones de mejora específicas sin alterar la disponibilidad de nutrimentos ni las propiedades del suelo.

\section{Agradecimientos}

A María Marta de la Salud Mora Matos y Juan Mejía Martínez, por su arduo trabajo al coadyuvar en la recolección de las muestras de suelo y tejido vegetal. A Mario Alberto Ortiz Jiménez por sus prácticas enseñanzas de estadística. 


\section{LiTERATURA CITADA}

Bodin Dresboll, D., J. Magid, K. Thorup-Kristensen. 2006. Long-Term Stability and Mineralization Rate Of Compost is Influenced by Timing of Nutrient Application During Composting of Plant Residues. Compost Science \& Utilization 14 (3): 215-221.

Boechat, C. L., J. A. Gonzaga Santos, A. M. de Aguiar Accioly. 2013. Net mineralization nitrogen and soil chemical changes with application of organic wastes with "Fermented Bokashi Compost ." Acta Scientiarum. Agronomy 35 (2): 257-264.

Bray, R. H., L. T. Kurtz. 1945. Determination of total, organic, and available forms of phosphorus in soils. Soil Science 59: 39-45.

Camacho-Tamayo, J. H., C. Luengas-Gómez, F. R. Leiva. 2010. Análisis multivariado de propiedades químicas en Oxisoles con diferentes niveles de intervención agrícola. Acta Agronómica 59 (3): 273-284.

Castañón-Nájera, G., L. Latournerie-Moreno, M. Mendoza-Elos, A. Vargas-López, H. Cárdenas-Morales. 2008. Colección y caracterización de Chile (Capsicum spp) en Tabasco, México. Phyton 77: 189-202.

Castro, A., C. Henríquez, F. Bertsch. 2009. Capacidad de suministro de N, P y K de cuatro abonos orgánicos. Agronomía Costarricense 33 (1): 31-43.

Contreras-Morales, E., G. Almaguer-Vargas, J. R. Espinoza-Espinoza, R. Maldonado-Torres, E. Álvarez-Sánchez. 2007. Distribución de Materia Seca y Nutrimentos en Árboles de Límon 'Persa' (Citrus latifolia Tan.) en Veracruz, México. Revista Chapingo. Serie Horticultura 13 (1): 77-85.

Etchevers Barra, J. D. 1999. Técnicas de diagnostico útiles en la medición de la fertilidad del suelo y el estado nutrimental de los cultivos. TERRA Latinoamericana 17: 209-219.

Gallardo, A., F. Covelo, L. Morillas, M. Delgado. 2009. Ciclos de nutrientes y procesos edáficos en los ecosistemas terrestres : especificidades del caso mediterráneo y sus implicaciones para las relaciones suelo-planta. Ecosistemas 18 (2): 4-19.

Grigatti, M., M. E. Giorgioni, S. Pilotti, C. Ciavatta. 2012. Stability, nitrogen mineralization capacity and agronomic value composto-based growing media for lettuce cultivation. Journal of Plant Nutrition 35: 704-725.

Hussain, S. S., T. Ara, F. A. Raina, G. Gani, N. Hussain, M. Hussain, S. R. Dar. 2015. Quality Evaluation of Different Forms of Compost and Their Effect in Comparison with Inorganic Fertilizers on Growth and Yield Attributes of Wheat (Triticum aestivum L.). Journal of Agricultural ScienceJournal of Agricultural Science 7 (1): 154-160.

Julca-Otiniano, A., L. Meneses-Florián, R. Blas-Sevillano, S.
Bello-Amez. 2006. La materia orgánica, importancia y experiencias de su uso en la agricultura. IDESIA 24 (1): 49-61.

Maldonado T. R., V. G. Almaguer, S. E. Robledo. 2008. Diagnóstico nutrimental y validación de dosis de fertilización para limón 'Persa'. TERRA Latinoamericana 26 (4): 341-349.

Maldonado T R., V. G. Almaguer, S. M. E. Álvares, S. E. Robledo. 2008. Diagnóstico Nutrimental y Validación de Dosis de Fertilización para Limón 'Persa'. Terra Latinoamericana 26 (4): 341-349.

Mattos Junior, D., T. F. Milaneze, F. Alves Azevedo, J. A. Quaggio. 2010. Soil Nutrient Availability and its Impact on fruit Quality of Tahiti Acid Lime. Rev. Bras. Frutic. Jaboticabal 32 (1): 335-342.

Servicio de información agropecuaria y pesquera. 2017. Disponible en: http://infosiap.siap.gob.mx/aagricola_ siap_gb/ientidad/index.jsp (consultado el 21 de mayo de 2017).

Walkey, A., I. A. Black. 1947. An examination of the degthareff method for determining soil organic and a proposed modification of chromic acid titration method. Soil Science 37: 29-38. 